\section{NOVA TELLVS}

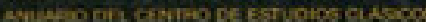

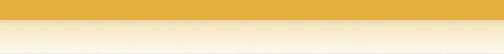

\section{Nova Tellus}

ISSN: 0185-3058

novatelu@servidor.unam.mx

Centro de Estudios Clásicos

México

Quiñones Melgoza, José

Dedicatoria de Cristóbal Cabrera a Fray Juan de Zumárraga, obispo de México

Nova Tellus, vol. 29, núm. 1, 2011, pp. 335-346

Centro de Estudios Clásicos

Distrito Federal, México

Disponible en: http://www.redalyc.org/articulo.oa?id=59121302017

- Cómo citar el artículo

Número completo

- Más información del artículo

Página de la revista en redalyc.org

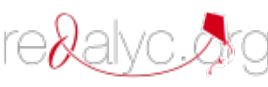

Sistema de Información Científica

Red de Revistas Científicas de América Latina, el Caribe, España y Portugal

Proyecto académico sin fines de lucro, desarrollado bajo la iniciativa de acceso abierto 


\title{
Dedicatoria de Cristóbal Cabrera a Fray Juan de Zumárraga, obispo de México
}

\author{
José QuiÑones MeLGOZA \\ Universidad Nacional Autónoma de México \\ jqmelgoza@yahoo.com.mx
}

RESUMEN: La carta, dirigida a Fray Juan de Zumárraga, da a conocer algunas notas biográficas de Cristóbal Cabrera: su ordenación sacerdotal, su traducción del griego al latín a los argumentos de todas las Epístolas de san Pablo y otras canónicas de la Biblia; además, que vino a la ciudad de México con don Vasco de Quiroga a la Junta de Obispos (1539) y que estuvo hospedado en la casa de virrey.

\section{Cristóbal Cabrera's letter dedicated to Fray Juan de Zumárraga, bishop of Mexico}

\begin{abstract}
This document refers to the letter that Cristóbal Cabrera addressed to Fr. Juan de Zumárraga, which contains some biographical important news, such as: his ordainment, his translation, from Greek to Latin, of the arguments to all of St. Paul's epistles and other canonical ones of the Bible; also, that along with Vasco de Quiroga, he attended the Meeting of Bishops in Mexico City (1539) and that he was hosted by the viceroy himself.
\end{abstract}

Palabras Clave: Cristóbal Cabrera, Fray Juan de Zumárraga, Vasco de Quiroga, Epístolas de San Pablo.

KEYwORDs: Cristóbal Cabrera, Fray Juan de Zumárraga, Vasco de Quiroga, Saint Paul's Epistles. FECHA DE RECEPCIÓN: 25 de febrero de 2011.

FECHA DE ACEPTACIÓN: 28 de mayo de 2011. 



\title{
Dedicatoria de Cristóbal Cabrera a Fray Juan de Zumárraga, obispo de México
}

\author{
José QuiÑones MELGOZA
}

Efectivamente Cristóbal Cabrera ${ }^{1}$ colocó, antes de su trabajo de traducción y después de su título, Argumenta in omnes Beati Pauli Epistolas et alias Catholicas ac canonicas e Graeco in Latinum versa per Christophorum a Iesumaria (cum adolescens adhuc apud Novae Hispaniae Indos ageret), esa dedicatoria. En cuanto al título de la obra que dedicó al obispo Fray Juan de Zumárraga, Elisa Ruiz ${ }^{2}$ refiere que "Hay correcciones en el título de la obra. La frase del encabezamiento que hemos puesto entre paréntesis quizás sea una adición. Tras Iesumaria había, tal vez [la palabra] sionius (sic) que, posteriormente, ha sido borrada por el autor. Este adjetivo [a Iesumaria, a modo de patronímico] que se lee en [párrafo $1 \mathrm{de}$ ] la cartadedicatoria dirigida a Zumárraga ha sido igualmente suprimido".

Ernest J. Burrus, que reproduce la dedicatoria ${ }^{3}$ y que la titula "Cabrera's letter to the bishop of Mexico, Juan de Zumárraga: complete text of the original", informa que el autógrafo personal se guarda en la Biblioteca Vaticana, manuscrito latino 1164 (año 1540 y 1559) ff. 173-188v, y que éste no se halla mencionado en la Bibliotheca Hispana Nova de Nicolás Antonio ni (hasta la fecha de su artículo) en otra bibliografía impresa; pero Elisa Ruiz, citando el mismo manuscrito y los mismos años, señala diferentes fojas (263-278), y dice que la fecha de 1559 debe aplicarse a la

\footnotetext{
${ }^{1}$ Cristóbal Cabrera nació en 1513 en la provincia de Burgos, España. Llegó a la ciudad de México cerca de 1534, siendo tal vez clérigo de órdenes menores, pues Nicolás Antonio dice que "se dedicó a una vida célibe y eclesiástica". Aquí fue acogido por el obispo Fray Juan de Zumárraga, quien lo ordenó sacerdote en 1535, a la edad de 22 años. Vivió en Nueva España más o menos 12 años, ya que para 1545 se encuentra de regreso en su patria, de donde parte para Roma, y allá muere en 1598.

2 “Cristóbal Cabrera, apóstol grafómano", Cuadernos de filología clásica, 12 (Madrid, 1977), p. 81-82.

3 "Cristóbal Cabrera (c.1515-1598) first American Author: A check list of his Writings in The Vatican Library", Manuscripta, 4 (Saint Louis, 1960), pp. 85-89. Ignoro por qué el padre Burrus (que fue el primero) llama carta a la dedicatoria. Los posteriores siguen su opinión no bien fundada, porque Cabrera dice (parágrafo 24): Hoc ergo quicquid est laboris mihi visum est... tuae nuncupare prudentiae (Por tanto lo que sea este trabajo, me pareció dedicarlo a tu competencia).
} 
disposición de la obra Sancti Evangelii Quadriga, escrita por propia mano de Cabrera en Medina de Ríoseco, ${ }^{4}$ la cual va encuadernada en el mismo volumen (manuscrito 1164).

Leopoldo Campos ${ }^{5}$ también reprodujo la dedicatoria con el título de "Carta de Cristóbal Cabrera, Pbro., a Fr. Juan de Zumárraga obispo de México". Antes de editarla, señala en la nota 33: "Aun cuando el P. Burrus edita dicha carta... nosotros hacemos otro tanto, aprovechando la copia que desde hace algún tiempo nos obsequió el notable americanista y hermano en Religión P. Lino Gómez Canedo, O.F.M., quien la sacó de su puño y letra durante sus investigaciones en Roma, y a quien agradecemos públicamente su generosidad". ${ }^{6}$

Tomando como base esas dos ediciones, efectuadas hace 50 y 38 años respectivamente, me puse a realizar (sin tener en mis manos el manuscrito original) esta edición crítica, en la cual sigo para su correspondiente grafía las normas comunes que los filólogos más connotados utilizan en las ediciones de los textos clásicos latinos. ${ }^{7}$ Sobre todo tomé en cuenta el empleo reglamentario de mayúsculas y minúsculas al inicio de nombres propios o comunes, o bien corregí la deformación gráfica de muchas voces, propiciada por el descuido de latines, como el vulgar, el bíblico-eclesiástico y el usado en los escritos patrísticos.

Tocante a la puntuación, conservo, omito o cambio la que consignan los textos que seguí en mi trabajo. Ella atiende a la estructura y modismos de la lengua latina y a su sintaxis, ya que busqué hacer más comprensible el texto de Cabrera. Sólo copio tal cual la puntuación que lleva, en la edición de la Vulgata que manejé, ${ }^{8}$ la cita bíblica que el autor de la Dedicatoria hace del profeta Ezequiel (parágrafos 3-6), la cual pongo con sus mismos párrafos, ya que Cabrera los alteró, y meto entre paréntesis angulares las palabras que éste añadió y entre corchetes la que suprimió del texto bíblico.

\footnotetext{
${ }^{4}$ Ruiz, op. cit., pp. 81 y 84.

5 "Métodos misionales y rasgos biográficos de Don Vasco de Quiroga según Cristóbal Cabrera, P[res]b[íte]ro", en Don Vasco de Quiroga y el Arzobispado de Morelia, Morelia, 1972, pp. 116-123.

${ }^{6} \mathrm{Ib} ., \mathrm{p} .116$.

${ }^{7}$ Entre otras, empleo la $\mathbf{v}$ para sonido consonántico y la u para vocálico. Transcribí la $\mathbf{j}$ con $\mathbf{i}, v$. gr.juvenes por iuvenes.

${ }^{8}$ Biblia sacra iuxta Vulgatam Clementinam. Nova ed. logicis partitionibus aliisque subsidiis ornata a Alberto Colunga et Laurentio Turrado, 4a. ed. Matriti, 1965 (BAC, 14), p. 835.
} 
Por razones prácticas, pedidas por el aparato crítico, divido el texto de la Dedicatoria en parágrafos que, como añadidos míos, van puestos con corchetes negritos y añado aquí aquellas notas que ayudan a apreciar mejor al autor y a su documento.

Hac itur ad astra... (parágrafo 8). Alude a la Eneida de Virgilio, IX, 641: Sic itur ad astra.

Nos... nonne sumus numerus fruges consumere nati? (parágrfo 8). Alude a Epistolas de Horacio, I, II, 27: Nos numerus sumus et fruges consumere nati.

Editione Complutensi... (parágrafo 9). Se refiere a la Biblia políglota en 5 tomos, edición auspiciada por el cardenal Cisneros, y publicada en Alcalá de Henares, 1502-1514, de la cual existe un ejemplar en la Biblioteca Nacional de México.

Michuacanensis episcopus... (parágrafo 11). Es don Vasco de Quiroga, primer obispo de Michoacán.

Apud proregem... (parágrafo 11). Es el virrey Antonio de Mendoza, que en 1534 vino a Nueva España como presidente de la Segunda Audiencia, la cual también integraba don Vasco de Quiroga.

Synodali... Consilio... (parágrafo 11). Fue la Junta Episcopal (o Sínodo de obispos) que se inició en la ciudad de México el 27 de abril de 1539 y a la cual asistieron, entre otros prelados: Zumárraga, Quiroga, Zárate, Garcés y Marroquín, y Cabrera acompañaba a Quiroga, por eso fue hospedado también en la casa del virrey.

Has mihi bovi... imponi clitellas... (parágrafo 12). Se hace referencia al proverbio antiguo: bovi clitellas imponere que $A$ New Latin Dictionary (Lewis and Short). Bajo la palabra bos dice que se aplicaba a quien desempeñaba una tarea para la cual no era competente. Cf. Cicerón, $A d$ Att., V, XV, 3: Clitellas bovi sunt impositae...

Exemplari Basiliensis... (parágrafo 13) Burrus dice que se refiere a la traducción del griego que Erasmo hizo del "Nuevo Testamento". Hasta 1539 se habían hecho de ella cinco ediciones $(1516,1519,1522,1527$ y 1535).

Finalmente las siglas en el aparato crítico designan: B, la edición de Burrus; C, la edición de Campos, a las cuales se añadió ER, Elisa Ruiz porque dentro de su estudio reproduce el título y algunos fragmentos de la Dedicatoria. ${ }^{9}$

\footnotetext{
${ }^{9}$ Son los parágrafos 9, 13-14 y 24-25, que ella reproduce en las pp. 63 y 82 (op. cit.).
} 
[1] Reverendissimo in Christo patri Ioanni Zumarragae, episcopo primo dein archiepiscopo Mexicensis Ecclesiae in Nova Hispania, detestabilium hereseon inquisitori, Christophorus a Iesumaria salutem in Domino sempiternam.

[2] Si, iuxta rhetorum sententiam, praecipua pars orationis in auditoribus sita est (quippe quod frustra videatur quispiam dicere, nisi auditorem habeat attentum, docilem et benevolum), hic non possum non agere tecum paucis, amplissime praesul, quod sciam te variis ubiquaque premi distringique negotiis, dum vere episcopum, hoc est, speculatorem vigilantissimum agis. [3] Nimirum meministi illud Ezechielis [XIII, 6-9]:

Si speculator < ait Dominus> viderit gladium venientem, et non insonuerit buccina, et populus se non custodierit, veneritque gladius, et tulerit de eis animam: ille quidem in iniquitate sua captus est, sanguinem autem eius de manu speculatoris requiram.

[4] Et tu, fili hominis, speculatorem dedi te domui Israel; audiens ergo ex ore meo sermonem, annuntiabis eis ex me. [5] Si me dicente ad impium: [Impie,] morte morieris, non fueris locutus ut se custodiat impius a via sua, ipse impius in iniquitate sua morietur, sanguinem autem eius de manu tua requiram.

[6] Si autem annuntiante te ad impium ut a viis suis convertatur, non fuerit conversus a via sua, ipse in iniquitate sua morietur, porro tu animam tuam liberasti.

[7] Hinc est quod tam vigilans innumeris laboribus incumbas, quibus hinc inde impetitus. Dum alii bis pransi cenam parant, tu prandium primum attingas, ut iam cum Apostolis et Apostolorum duce Christo spatium tibi deest manducandi. Dumque alii dormitum eunt, tu ad scribendum his et illis tuas ecclesias moderantibus, insomnes ducens noctes, te recipias. Perge, pie praesul, perge, inquam, qua coepisti currere.

[8] Hac itur ad astra, ut ille dixit. Nos homunculi ad te tuique similes huius Novi Orbis praesules sic in laboribus assiduis collati, nonne sumus numerus fruges consumere nati?

[9] Sed ad rem. Religiosi quidam viri ex instituto divi Dominici ut morum probitate singularique eruditione insignes, ita mihi non vulgares amici superioribus a me diebus obnixe petierunt ut Argumenta illa in Divi Pauli Epistolas et ceteras catholicas ac canonicas cum nonnulis aliis quae Graece ferebantur in editione Complutensi facerem Latina. [10] Detrectaram semel atque iterum hanc sarcinam, quod esset meis humeris longe maior et plerisque tricis implicitus carerem etiam hos- 
pitio studiorum silentiis accomodo; nam eo nido hospes sum Mexici ubi nihil non est obstreperum, turbulentum ac clamosum, vulcanicam officinam dicas rectius quam litterariam.

[11] Haec invitus patior in aula, ferendum tamen, quod pius itidem Michuacanensis episcopus hospes hic apud proregem patitur, ne tantum patronum deseram, donec Synodali absoluto Concilio, hinc exeamus. [12] Illi vero quod identidem efflagitarunt, impetrarunt tandem; et passus sum volens nolens has mihi bovi (ut est in ethnicorum proverbiis) imponi clitellas.

[13] Accipiens igitur oblatum codicem, memet in Museum sine Musis recipio. Et, ubi primum singula quaeque eorum quae traducturus eram inspexi, inveni eadem fere omnia in exemplari Basiliensi (cuius copia nobis fecerat caritas tua), recentioribus elegantioribusque typis excussa. [14] Itaque ex utriusque exemplaris collatione, Complutensis scilicet et Basilensis, utcumque verti Argumenta illa Graeca quae in omnes Novi Testamenti Epistolas traduntur anonyma, id est incerto autore. Atqui nemo ob id aspernatu digna haec ipsa censeat. [15] Nec enim extarent adhunc tamquam sacrosanctum quoddam in Novi Testamenti adytis reconditum nec in pretio forent eruditis habita, nisi prae se ferrent auctorem, qui nomen forte modestiae gratia supprimere voluit, doctissimum gravissimumque virum. [16] Quod pariter accidisse videtur in nostris illis Argumentis quae a priscis recepta Latinis patribus citra ullam auctoris nomenclaturam hodie leguntur in Bibliis. [17] Quando res ipsa per se est optima, aut certe honesta simul et utilis, quid vetat, quominus illam obviis amplectamur ulvis, etiamsi ipsum ignoremus artificem? [18] Etenim, qui ab institore decoris lineamentis pictam sculptamque imaginem velit emere, parum sit anxius decet de notitia nomineque pictoris aut sculptoris. [19] Perparum enim refert scire sitne Flandrus an Gallus, Germanus an Italus.

[20] Alia insuper Argumenta ex Complutensi exemplari sub Theodoriti nomine in omnes ferme quas memoravi Epistolas breviora quidem illa sed lectu maxime digna transtuli. Adde his Peregrinationem Beati Pauli cum ratione temporis praedicationis ac martyrii eiusdem Apostoli. [21] Quod in Basiliensi editione coniuncte positum erat incerto auctore, in Complutensi vero Peregrinatio Pauli distincta erat, nulla mentione facta de auctore, ab aliis quae sub Euthalii diaconi nomine ferebantur. [22] Postremo Epistolam eruditissimi viri Eusebii Caesariensis ad Carpianum de Evangelistarum concordia quae argumenti vice est in Canones eiusdem viri Latinam feci. 
[23] Ceterum fidelem potius quam de elegantia sollicitum egi interpretem. Idque data opera ne in re tam simpliciter sancta stulte penuriam meam ostentare viderer.

[24] Hoc ergo quicquid est laboris mihi visum est, secundum Deum cui me totum debeo, tuae nuncupare prudentiae. [25] Nam praeter quam quod me quondam pene puerum statim ex Hispania ingressum Mexicum domi fovisti, aluisti ac humanitater reparasti amoreque vere paterno semper es prosecutus, donasti etiam hypodiaconi, diaconique munere; ac demum Christi gratia actum est ut modo mihi indigno imbecillique adolescenti presbyterii onus et honos imponeretur. [26] Reliquas autem animi tui dotes quas a Patre luminum accepisti, prudens sciensque praetereo, ut adulationis notam subterfugiam.

[27] At cum Deo optimo maximo omniumque liberalissimo remuneratore tibi res est. Ipse multo cum fenore quod seminas tibi reddet et cum beatis pro meritis mercedem recipies. [28] Quod superest, tuae erit charitatis conatus nostros boni consulere Christophorique tui studiis favere.

[29] Praestiti quidem quod pro virili potui, si minus contigit quod volui. [30] Porro si sensero tuam amplitudinem meam probavisse industriam, ut omittam quod ad alia reddar alacrior, spes erit fore ut nemo lectorum eam audeat improbare. [31] Illud interim impetrare velim a criticis, videlicet ut priusquam suam proferant sententiam, perpendant me nihil hic addidisse de meo, nisi cum animi sinceritate laborem. [32] Nam, ut ad doctos me convertam, quis adeo tetricus est, aut livore corruptus, aut lippis oculis caecutiens, ut non videat haec quantulacumque sunt, non parum lucis allatura studiosis lectoribus, sacrarumque literarum candidatis, illisque potissimum Graecanicae linguae ignaris, in quorum gratiam haec Latinis auribus reddita sunt?

[33] Sed ne sim aequo longior, bene valeat caritas tua in Christo Iesu.

[34] Mexici. Ad festum Omnium Sanctorum, anno a virgineo nostrae salutis partu 1540 .

\section{Aparato crítico}

1 patri... episcopo... archiepiscopo... inquisitori: Patri... Episcopo... Archiepiscopo... Inquisitori B C.II $\mathbf{2}$ sita est (quippe... benevolum): sita est -quippe... benevolum- $\mathrm{B}$; sita est, quippe... benevolum, C; auditorem: auditorium $\mathrm{C}$ (cf. supra parágrafo 2: auditoribus); praesul: Praesul 
B C; ubiquaque: undiquaque $\mathrm{B}$; undequaque C.II $\mathbf{3}$ buccina: bucina $\mathrm{C}$; sanguinem: sanguine B; autem eius de manu: autem de manu B.ll 7 cenam: caenam B C; praesul: Praesul B.II 8 assiduis: assiduos B C.II 9 Dominici ut morum: Dominici morum C; ceteras: caeteras C; catholicas ac canonicas: Catholicas ac Canonicas C; Graece: grece C; Latina: latina C; Latino ER.II 10 Detrectaram: Detrectarem C; litterariam: literariam B.ll 11 Michuacanensis: Mechuacanensis C; episcopus: Episcopus B C; proregem: Proregem B C.II 13 Museum sine Musis: musaeum sine musis B C ER; caritas: Charitas B C.II $\mathbf{1 4}$ exemplaris: exemplari C.II $\mathbf{1 5}$ tamquam: tamque B, auctorem: authorem B.ll $\mathbf{1 6}$ patribus: Patribus $\mathrm{C}$; Bibliis: Bibliis B; Biblis C.II 17 Quando res ipsa per se est optima: Quando res est ipsa per se optima C.II $\mathbf{2 1}$ incerto auctore, in Complutensi vero Peregrinatio Pauli. Hoc totum deest in C; auctore: autore B; distincta erat: distinctum erat C.II 23 Ceterum: caeterum B C.II 24 prudentiae: Prudentiae B C.II 25 quondam: quodam B C ER; honos: honor C.II 27 fenore: foenore B C.II 28 caritatis: Charitatis B C.Il 29 amplitudinem: Amplitudinem B.II $\mathbf{3 3}$ caritas: Charitas B C; Iesu: Jesu C.

[1] Cristóbal de Jesús María saluda por siempre en el Señor al reverendísimo padre en Cristo Juan de Zumárraga, primer obispo y después arzobispo de la Iglesia de México en Nueva España e inquisidor de abominables herejías.

[2] Si, según la enseñanza de los retórico, la parte principal de un discurso descansa en los oyentes (por cierto parecerá que alguien habla en vano, si no tiene un oyente atento, dócil y benévolo), aquí puedo hablar brevemente contigo, máximo obispo, porque sé que tú por todas partes estás oprimido y ocupado por varios negocios, mientras verdaderamente vives como obispo, esto es, vigilantísimo atalaya. [3] Seguramente recordaste aquello de Ezequiel:

Si el atalaya <dice el Señor> viera que un ejército viene y no hiciera sonar la trompeta y el pueblo no se protegiera y viniera el ejército y les quitara la vida, él ciertamente estará cautivo en su maldad; sin embargo, yo del cuidado del atalaya reclamaré esa sangre.

[4] Y tú, hijo de hombre, te elegí atalaya de la casa de Israel. Así, pues, cuando, oigas de mi boca el mandato se lo comunicarás por mí. [5] Si diciéndole yo al impío: [Impío,] morirás en tu maldad y tú no hablaras para que el impío se proteja de su camino, el mismo impío morirá en su maldad; sin embargo, yo reclamaré su sangre de tu cuidado. 
[6] Mas si comunicándoselo tú al impío para que de sus caminos se convierta, y no fuera convertido de su camino, él mismo morirá en su maldad, pero tú libraste tu alma.

[7] De un lado está que tan vigilante te consagres a innumerables trabajos; del otro, que algunos te censuren. Mientras unos dos veces desayunados preparan la comida, tú pruebas el primer desayuno, tal como ya pasó con los Apóstoles y con Cristo, guía de los Apóstoles, te falta tiempo para comer. Y mientras otros van a dormir, tú te recoges a escribir a éstos y a aquellos que gobiernan tus iglesias, pasando insomne las noches. Prosigue, piadoso obispo, prosigue, insisto, por donde comenzaste a correr.

[8] Por aquí se va a los astros, como dijo Virgilio. Yo, pobre hombre junto a ti y parecido a ti, obispo de este Nuevo Mundo, tan aplicado a asiduos trabajos, ¿no soy acaso sólo número nacido para consumir frutos?

[9] Pero voy a mi asunto. Ciertos religiosos de la orden de Santo Domingo, tan insignes en buenas costumbres y singular erudición como para mí distinguidos amigos, en días pasados me pidieron encarecidamente que pusiera en latín Los argumentos de las Epístolas de San Pablo y las demás católicas y canónicas con algunas otras que circulaban en griego dentro de la edición complutense. [10] Una y otra vez había rechazado tal carga, porque era muy superior a mis fuerzas, y metido en muchísimas dificultades carecía también de un alojamiento apropiado para el silencio de los estudios, ya que estoy alojado en esa mansión de México, donde todo es ruidoso, turbulento y ensordecedor que más bien lo llamarías una fragua que un taller literario. [11] Contrariado sufro esto en mi aposento; sin embargo debo soportar lo que igualmente soporta el piadoso obispo de Michoacán, alojado aquí en casa del virrey, para no dejar a tan gran patrono hasta que, una vez terminado el Concilio Sinodal, salgamos de aquí. [12] Sin embargo ellos muchas veces me lo pidieron vivamente y por fin lo consiguieron; y padecí, queriendo y no queriendo, que me impusieran (como está en los proverbios de los paganos), como al buey, las albardas.

[13] Por tanto, recibido el ofrecido códice, yo mismo me recluí en un Museo sin Musas. Y, luego que examiné cada uno de los argumentos que iba a traducir, encontré los mismos, casi todos, en un ejemplar de Basilea (cuya copia me había facilitado tu caridad), impresos con tipos más modernos y elegantes. [14] Y así, de la confrontación de ambos 
ejemplares, es decir, el complutense y el de Basilea, de cualquier modo traduje los argumentos griegos que corren anónimos, esto es, de autor incierto, en todas las epístolas del Nuevo Testamento; sin embargo, nadie por esto los juzgue dignos de desprecio. [15] Y en efecto, no quedarían hasta ahora como algo sacrosanto, oculto en los santuarios del Nuevo Testamento, ni serían juzgados con aprecio por los eruditos, si frente a sí no llevaran por autor a un sujeto muy docto y notable que tal vez por modestia quiso suprimir su nombre. [16] Parece que esto sucedió igualmente en aquellos Argumentos nuestros que recibidos por los antiguos padres latinos sin ningún nombre de autor, hoy se leen en las Biblias. [17] Cuando un objeto por sí mismo es óptimo, o ciertamente honesto al par que útil, ¿qué impide que lo acojamos con afables brazos, aunque ignoremos a su propio artesano? [18] Así, pues, quien, por sus pinceladas de belleza, quiere comprar del vendedor una imagen pintada o esculpida, es normal que esté poco ansioso de saber y conocer el nombre del pintor o del escultor. [19] Muy poco, pues, le interesa saber si es flamenco, francés, alemán o italiano.

[20] Traduje además algunos Argumentos tomados del ejemplar complutense, puestos bajo el nombre de Teodoreto, para casi todas las Epístolas que mencioné: son ciertamente más breves, pero muy dignos de ser leídos. Añade a éstos la Peregrinación de San Pablo con la relación del tiempo de predicación y martirio del mismo Apóstol. [21] Lo que en la edición de Basilea había sido puesto juntamente con el incierto autor; en la complutense, por el contrario, la Peregrinación de Pablo era distinta, no haciendo ninguna mención sobre el autor, de otras que se presentaban bajo el nombre del diácono Eutalio. [22] Finalmente traduje al latín la Epístola del muy erudito autor Eusebio de Cesarea a Carpiano sobre la concordancia de los Evangelistas, la cual tiene, en vez del argumento, los Cánones del mismo autor.

[23] Por lo demás, traduje como fiel intérprete, más que preocupado por la elegancia. Y esto lo hice, recibida la obra, porque en una cosa tan sencillamente santa, no pareciera que yo mostraba tontamente mi falta de talento.

[24] Por tanto lo que sea este trabajo, me pareció, según Dios, a quien todo me debo, dedicarlo a tu competencia. [25] Pues además de que otrora, casi niño, tan pronto como entré desde España a la ciudad de México, me acogiste en tu casa, me alimentaste y humanamente me alentaste de nuevo, y con amor verdaderamente paternal me honraste 
siempre; me diste también el subdiaconado y el diaconado, y finalmente por la gracia de Cristo se procuró que en seguida por ti me fuera impuesta (indigno y débil adolescente) la honrosa carga del sacerdocio. [26] Sin embargo para eludir la mancha de la adulación, deliberada y conscientemente paso por alto las demás dotes de tu alma, que recibiste del Padre de las luces.

[27] No obstante tienes que vértelas con Dios todopoderoso y remunerador muy pródigo de todas las cosas. Él mismo te devolverá con mucha ganancia lo que siembras y por tus méritos recibirás la recompensa en compañía de los santos. [28] Por lo demás será propio de tu caridad acoger bien mis esfuerzos y favorecer los estudios de tu Cristóbal.

[29] Ciertamente realicé lo que individualmente pude, aunque no logré lo que quise. [30] Pero si siento que tu dignidad aprobó mi diligencia, para omitir lo que para otras cosas traduzca más alegre, tendré venidera esperanza de que nadie de los lectores se atreverá a reprobarla. [31] Entre tanto, esto quisiera pedir de los críticos: que, antes que divulguen su opinión, sopesen bien que yo en ello nada añadí de lo mío, sino un trabajo con pureza de intención. [32] Pues, para dirigirme a los doctos, ¿quién será tan severo, roído de envidia o ciego en sus ojos legañosos que no mire que estos Argumentos, por poco que valgan, llevarán no poca luz a los lectores estudiosos y a los aspirantes a estudiar las Sagradas Escrituras, y principalmente a los ignorantes de la lengua griega, en beneficio de cuyas orejas latinas estos fueron traducidos?

[33] Pero para no alargarme más de lo justo, bien haya en Jesucristo tu caridad.

[34] En México. Para la fiesta de todos los santos, en el año de 1540 del parto virginal de nuestra salvación. 